\title{
ORIGINAL RESEARCH \\ Improved Detection of Skull Metastasis with Diffusion-Weighted MR Imaging
}

\author{
A.J. Nemeth \\ J.W. Henson \\ M.E. Mullins \\ R.G. Gonzalez \\ P.W. Schaefer
}

BACKGROUND AND PURPOSE: Metastasis to the skull is clinically important, but routine MR imaging offers moderate sensitivity for skull-metastasis detection in our experience. We sought to determine if diffusion-weighted MR imaging (DWI) could improve the detection of skull metastasis in patients with primary carcinomas that metastasized to bone compared with conventional MR imaging.

MATERIALS AND METHODS: Seventy-five patients from the tumor registry of our institution with extracranial primary malignancy who had brain MR imaging with DWI and radionuclide bone scanning (RNBS, gold standard) within a 6-week interval were evaluated. Thirty-eight patients demonstrated increased radiopharmaceutical uptake on RNBS, consistent with skull metastasis of any size, and the remaining 37 were control subjects. Two readers correlated the DWI and conventional MR imaging with RNBS.

RESULTS: The overall sensitivity of DWI for detection of skull metastases was $68.4 \%-71.1 \%(\kappa=$ $0.68)$ versus $42.1 \%-55.3 \%(\kappa=0.65)$ for conventional MR imaging. Breast cancer $(n=20)$ was detected with greatest sensitivity of $86.7 \%-93.3 \%(\kappa=0.80)$ for DWI versus $60 \%-80 \%(\kappa=0.5)$ for conventional MR imaging. Lung cancer $(n=32$ ) was detected with $63.6 \%-72.7 \%$ sensitivity $(\kappa=$ $0.56)$, and prostate cancer $(n=8)$ with $14.3 \%$ sensitivity $(\kappa=0.5)$ for DWI versus $27.3 \%-36.4 \%(\kappa=$ $0.81)$ and $14.3-42.9 \%(\kappa=0)$, respectively, for conventional MR imaging.

CoNCLUSIONS: DWI is a useful sequence for identifying focal skull metastases for breast and lung malignancies and, compared with conventional MR imaging, provides improved detection of these lesions. DWI is insensitive for detecting skull metastases from prostate carcinoma.
D etection of skeletal metastasis is important in cancer staging and occurs most often in the presence of breast, lung, or prostate malignancy. The incidence of skull metastasis in a susceptible patient is high enough that a radiologist should be aware of its potential presence on brain imaging. Tofe et $\mathrm{al}^{1}$ showed a skull metastasis incidence rate of $23 \%$ in patients with breast, lung, or prostate cancer who underwent radionuclide bone scanning (RNBS), and the skull may be the only site of bony metastasis in up to $11.6 \%$ of patients. ${ }^{2}$ Due to the limited sensitivity of conventional MR imaging for skull metastasis in our experience, it is likely that these lesions are frequently missed in patients undergoing central nervous system (CNS) staging studies.

Diffusion-weighted MR imaging (DWI) is sensitive to the diffusion of protons and was used initially in the assessment of acute ischemic stroke. ${ }^{3,4}$ DWI has also been used for a wide range of other pathologies including metastasis to vertebral bodies, ${ }^{5-17}$ though there has been controversy on this subject. ${ }^{18,19}$ DWI may be helpful to differentiate pathologic compression fractures related to metastases from benign osteoporotic compression fractures. Histopathologic specimens have shown a different composition of bone marrow in metastatic

Received August 14, 2006; accepted after revision October 24.

From the Neuroradiology Division (A.J.N., J.W.H., R.G.G., P.W.S.) and Pappas Center for Neuro-oncology (J.W.H.), Massachusetts General Hospital, Harvard Medical School, Boston, Mass; the Departments of Radiology, Neurology, and Neurological Surgery (M.E.M.), Emory University Hospital, Atlanta, Ga; and the School of Medicine (M.E.M.), Emory University, Atlanta, Ga.

Paper previously presented at: Annual Meeting of the Radiological Society of North America, November 27-December 2, 2005, Chicago, III; and Annual Meeting of the American Society of Neuroradiology, May 6-12, 2006, San Diego, Calif.

Please address correspondence to Pamela W. Schaefer, MD, Neuroradiology Division, Massachusetts General Hospital, 55 Fruit St, Gray two Rm B-285, Boston, MA 02114; e-mail: pschaefer@partners.org

D0l 10.3174/ajnr.A0501 fractures so that attenuated infiltration of malignant cells results in decreased extracellular diffusion relative to bone marrow edema. ${ }^{8}$

We hypothesized that DWI may improve the detection of skull metastasis. Similar to the detection of metastasis in the spine, increased sensitivity may exist for the detection of a DWI hyperintense metastasis in the skull related to the normal hypointense background when compared with conventional MR imaging. We sought to determine if DWI could improve the detection of skull metastasis in patients with primary carcinomas that metastasize to bone.

\section{Materials and Methods}

\section{Patient Selection}

Approval was obtained from the institutional review board. We intended to find patients with skull metastasis who had a RNBS and DWI within a 6-week time interval for comparison. We reviewed the electronic medical records of 1000 patients of all ages, both male and female, with primary solid tumor outside the CNS, from the tumor registry of our institution between 1998 and 2004. Subjects were selected from these years so that all studies would be available on PACS. Inclusion criteria were the following: 1) RNBS (gold standard) included imaging of the skull; 2) brain MR imaging including DWI, T1-weighted imaging (T1WI) without and with gadolinium, fluidattenuated inversion recovery (FLAIR) imaging, and T2-weighted imaging (T2WI); 3) the above listed MR imaging sequences were performed within 6 weeks of RNBS.

From the tumor registry list, 38 patients had abnormal uptake in the skull on RNBS according to the nuclear medicine radiologist's report and met the 6-week interval criterion. Thirty-eight additional patients who met the inclusion criteria and who had no abnormal uptake on RNBS were included as control subjects. The study, therefore, began with 76 patients. However, 1 patient in the control group 
Table 1: DWI detection of RNBS-positive skull metastasis

\begin{tabular}{|c|c|c|c|c|c|c|}
\hline \multirow[b]{2}{*}{ Cancer Type } & \multicolumn{2}{|c|}{ Sensitivity } & \multicolumn{2}{|c|}{ Specificity } & \multirow[b]{2}{*}{$\kappa$} & \multirow{2}{*}{$\begin{array}{c}\text { Agreement Beyond } \\
\text { Chance of } \\
2 \text { Readers }\end{array}$} \\
\hline & $\begin{array}{c}\text { Reader } 1 \\
(\%)\end{array}$ & $\begin{array}{c}\text { Reader } 2 \\
(\%)\end{array}$ & $\begin{array}{c}\text { Reader } 1 \\
(\%)\end{array}$ & $\begin{array}{c}\text { Reader } 2 \\
(\%)\end{array}$ & & \\
\hline Overall, $n=75$ & $71.1(27 / 38)$ & $68.4(26 / 38)$ & $83.8(31 / 37)$ & $91.9(34 / 37)$ & $0.68(63 / 75)$ & Substantial \\
\hline Lung, $n=32$ & $72.7(8 / 11)$ & $63.6(7 / 11)$ & $76.2(16 / 21)$ & $85.7(18 / 21)$ & $0.56(25 / 32)$ & Moderate \\
\hline Breast, $n=20$ & $86.7(13 / 15)$ & $93.3(14 / 15)$ & $80(4 / 5)$ & $100(5 / 5)$ & $0.80(18 / 20)$ & Almost perfect \\
\hline Prostate, $n=8$ & $14.3(1 / 7)$ & $14.3(1 / 7)$ & $100(1 / 1)$ & $100(1 / 1)$ & $0.50(6 / 8)$ & Moderate \\
\hline Other, $n=15$ & $100(5 / 5)$ & $80(4 / 5)$ & $100(10 / 10)$ & $100(10 / 10)$ & $0.87(14 / 15)$ & Almost perfect \\
\hline
\end{tabular}

Note:-Other indicates genitourinary and gastrointestinal cancer, Ewing sarcoma, lymphoma, myxoid chondrosarcoma, squamous cell cancer of the head and neck, and thymic and undifferentiated cancer.

was later excluded on the basis that the conventional MR imaging was technically inadequate. Therefore, 75 patients were enrolled in the study.

\section{Imaging}

MR imaging was performed on a $1.5 \mathrm{~T}$ whole-body scanner. Using a 1.5T scanner, DWI was obtained by using single-shot echo-planar imaging with sampling of the entire diffusion tensor. Six high-b-value images corresponding to diffusion measurements in different-gradient directions were acquired followed by a single low-b-value image. The high b-value was $1000 \mathrm{~s} / \mathrm{mm}^{2}$, and the low b-value was 0 seconds/ $\mathrm{mm}^{2}$. Imaging parameters were a TR of 5 seconds, a TE of $90 \mathrm{~ms}$, an FOV of $22 \times 22 \mathrm{~cm}$, image matrix of $128 \times 128$ pixels, section thickness of $5 \mathrm{~mm}$ with a 1-mm gap, 23 axial sections, 5 signal intensity averages. Isotropic DWI was reviewed.

T1WI with and without gadolinium was acquired with a TR of 450 ms, effective TE of $20 \mathrm{~ms}$, FOV of $20 \mathrm{~cm}$, image matrix of $256 \times 256$ pixels, section thickness $5 \mathrm{~mm}$ with a $1-\mathrm{mm}$ gap, and 1 signal intensity average. Fast spin-echo T2WI was acquired with a TR of $5700 \mathrm{~ms}$, effective TE of $110 \mathrm{~ms}$, FOV of $20 \mathrm{~cm}$, image matrix of $512 \times 512$ pixels, section thickness of $5 \mathrm{~mm}$ with a $1-\mathrm{mm}$ gap, and 1 signal intensity average. FLAIR images were acquired with a TR of $9002 \mathrm{~ms}$, effective TE of $138 \mathrm{~ms}$, an FOV of $20 \mathrm{~cm}$, image matrix of $256 \times 256$ pixels, section thickness $5 \mathrm{~mm}$ with a $1-\mathrm{mm}$ gap, and 1 signal intensity average.

RNBS acquisition was performed 3 hours after the intravenous administration of $20 \mathrm{mCi}$ of technetium Tc99m methylene diphosphonate by a gamma camera with anterior and posterior whole-body acquisition of 2,700,000 counts for a 1200-second duration at a $256 \times$ 1024 image matrix with a 2.4-mm pixel size and spot images of the skull of 1,000,000 counts for a 260 -second duration at a $256 \times 256$ image matrix with a $2.4-\mathrm{mm}$ pixel size.

\section{Image Interpretation}

Two blinded readers each reviewed independently and in randomized order the DWI of each of the 75 patients in succession. The DWI was read as positive or negative for focal skull hyperintense signal and then correlated with the RNBS to confirm the position of the findings.

Subsequently, at a separate time point, each reader reviewed the conventional MR imaging of each of 75 patients, including T1WI without and with gadolinium, T2WI, and FLAIR imaging. The conventional MR imaging was read as positive or negative for a T1 hypointense, FLAIR, and T2 variable signal intensity (hypointense for prostate metastases but hyperintense for lung metastases) and enhancing skull lesions and then was correlated with RNBS to confirm the position of the findings. A kappa statistic was determined between the 2 readers. ${ }^{20}$

If the DWI or conventional MR imaging was read as abnormal in a different location than that in which the lesion was identified on the RNBS, the DWI or conventional MR imaging was recorded as a falsepositive. The lesions were quantified on a per-patient basis so that if the RNBS showed more than 1 lesion and the DWI or conventional MR imaging detected only 1 lesion, a positive result for DWI or conventional MR imaging was assumed.

\section{Data Analysis}

The sensitivity and specificity were determined for the DWI detection of RNBS-positive skull metastasis for both readers. The overall group was analyzed, and then each cancer type was analyzed. $\kappa$ was obtained for all groups to determine qualitatively the degree of agreement beyond chance for the 2 readers. ${ }^{20}$ This methodology was repeated for the conventional MR imaging detection of RNBS-positive skull metastasis for both readers. Then, the results of both DWI and conventional MR imaging studies were compared, including assessment for whether 1 of the imaging methods offered greater sensitivity for focalversus-diffuse metastases. To determine whether the sensitivity of DWI-versus-conventional images for the detection of bone metastases was statistically significantly different, we performed the McNemar test for comparison of success rates in paired data.

\section{Results}

The sensitivity and specificity of DWI for detecting skull metastasis for all types of primary malignancy were $68.4 \%-71.1 \%$ and $83.8 \%-91.9 \%(\kappa=0.68)$, respectively (Table 1$)$ (Fig 1$). \kappa$ was consistent with a substantial degree of agreement beyond chance between the 2 readers for the DWI detection of skull metastasis from all types of primary malignancy. For the gold standard RNBS, the $\kappa$ was 1 , consistent with perfect agreement.

When categorized by type of primary malignancy, breast cancer was detected on DWI of the skull with greatest sensitivity with a sensitivity and specificity of $86.7 \%-93.3 \%$ and $80 \%-100 \%$, respectively (Table 1 ). $\kappa$ showed an almost perfect degree of agreement between the readers for breast carcinoma $(\kappa=0.80)$. Lung cancer was detected on DWI with the next highest sensitivity with a sensitivity and specificity of $63.6 \%$ $72.7 \%$ and $76.2 \%-85.7 \%$, respectively, and $\kappa$ showed a moderate degree of agreement (0.56). DWI was least sensitive for detecting prostate metastasis (sensitivity of $14.3 \%$ and specificity of $100 \%)$ with $\kappa$ showing a moderate degree of agreement (0.50).

Fifteen of the 75 patients had less common primary tumors, 5 of whom had skull metastasis on RNBS and 10 of whom were control subjects (Table 1). Less common tumor types included genitourinary and gastrointestinal tumors, with 5 and 4 subjects respectively. Ewing sarcoma, lymphoma, 

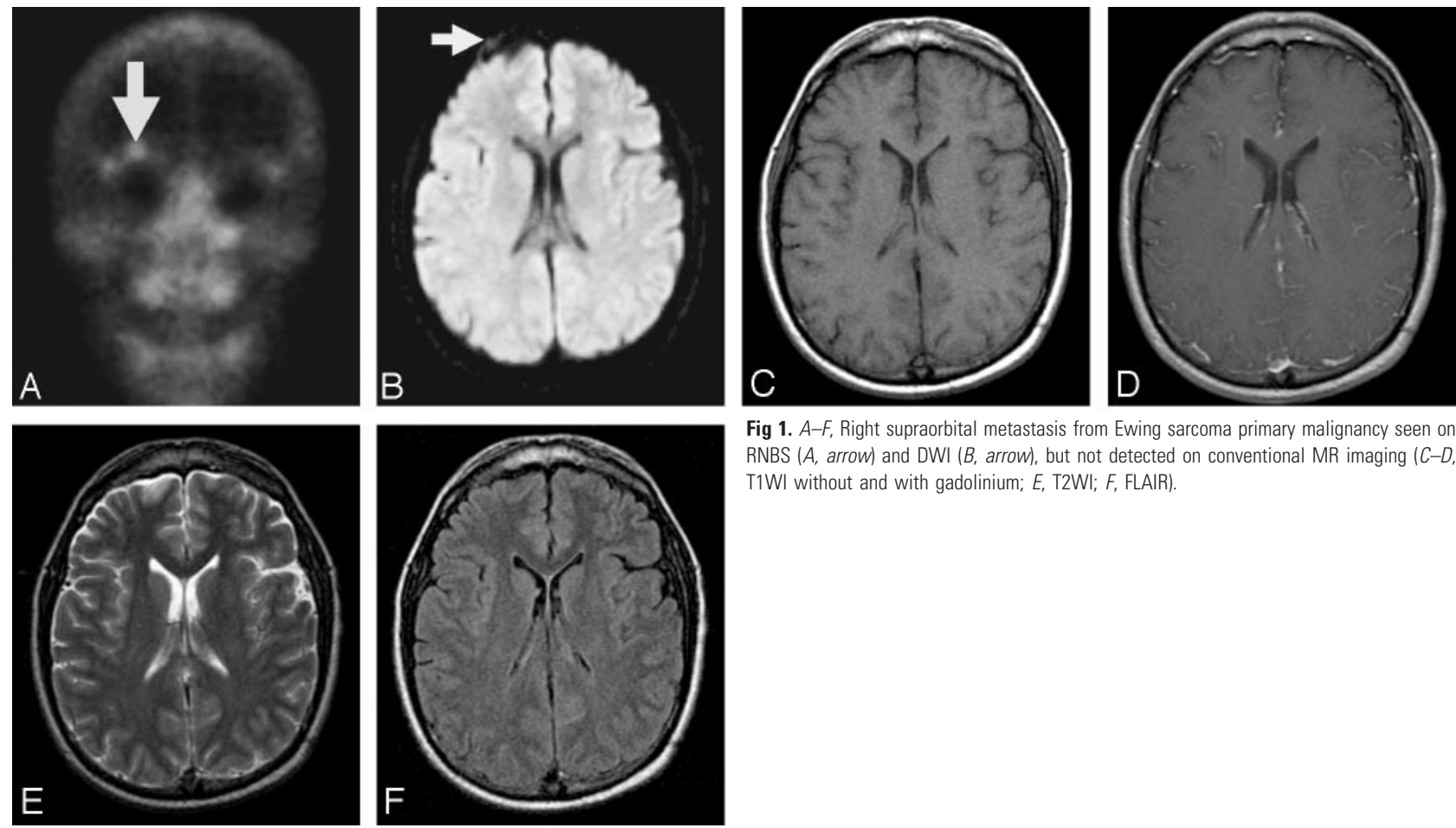

Fig 1. $A-F$, Right supraorbital metastasis from Ewing sarcoma primary malignancy seen on RNBS $(A$, arrow) and DWI $(B$, arrow), but not detected on conventional MR imaging $(C-D$, T1WI without and with gadolinium; $E$, T2Wl; F, FLAIR).

Table 2: Conventional MRI detection of RNBS-positive skull metastasis

\begin{tabular}{|c|c|c|c|c|c|c|}
\hline \multirow[b]{2}{*}{ Cancer Type } & \multicolumn{2}{|c|}{ Sensitivity } & \multicolumn{2}{|c|}{ Specificity } & \multirow[b]{2}{*}{$\kappa$} & \multirow{2}{*}{$\begin{array}{c}\text { Agreement Beyond } \\
\text { Chance of } \\
2 \text { Readers }\end{array}$} \\
\hline & $\begin{array}{c}\text { Reader } 1 \\
(\%)\end{array}$ & $\begin{array}{c}\text { Reader } 2 \\
(\%)\end{array}$ & $\begin{array}{c}\text { Reader } 1 \\
(\%)\end{array}$ & $\begin{array}{c}\text { Reader } 2 \\
(\%)\end{array}$ & & \\
\hline Overall, $n=75$ & $55.3(21 / 38)$ & $42.1(16 / 38)$ & $97.3(36 / 37)$ & $100(37 / 37)$ & $0.65(62 / 75)$ & Substantial \\
\hline Lung, $n=32$ & $27.3(3 / 11)$ & $36.4(4 / 11)$ & $100(21 / 21)$ & $100(21 / 21)$ & $0.81(29 / 32)$ & Almost perfect \\
\hline Breast, $n=20$ & $80(12 / 15)$ & $60(9 / 15)$ & $100(5 / 5)$ & $100(5 / 5)$ & $0.50(15 / 20)$ & Moderate \\
\hline Prostate, $n=8$ & $42.9(3 / 7)$ & $14.3(1 / 7)$ & $100(1 / 1)$ & $100(1 / 1)$ & $0(4 / 8)$ & None \\
\hline Other, $n=15$ & $60(3 / 5)$ & $40(2 / 5)$ & $90(9 / 10)$ & $100(10 / 10)$ & $0.93(13 / 15)$ & Almost perfect \\
\hline
\end{tabular}

Note:-Other indicates genitourinary and gastrointestinal cancer, Ewing sarcoma, lymphoma, myxoid chondrosarcoma, squamous cell cancer of the head and neck, and thymic and undifferentiated cancer.

myxoid chondrosarcoma, squamous cell cancer from the head and neck, and thymic and undifferentiated cancer were each represented by a single subject.

Comparison was then made between DWI and conventional MR imaging (Table 2) in the detection of skull metastases. The overall DWI sensitivity was improved relative to conventional MR imaging sensitivity for detecting skull metastases $(68.4 \%-71.2 \%$ versus $42.1 \%-55.3 \%)$, though the difference reached statistical significance with $P<.05$ only for reader 2 . There was a substantial degree of agreement between the readers for both the DWI $(\kappa=0.68)$ and conventional MR imaging $(\kappa=0.65)$ analysis.

The sensitivity for detecting breast and lung cancer was increased by using DWI relative to conventional MR imaging (86.7\%-93.3\% versus 60\%-80\% for breast cancer and 63.6\%$72.7 \%$ versus $27.3 \%-36.4 \%$ for lung cancer), whereas for prostate cancer, conventional MR imaging was more sensitive than DWI for 1 of the readers. The other reader detected prostate metastasis with equally low sensitivity for both DWI and conventional MR imaging.

For the less common tumor types, DWI increased the sensitivity of skull metastasis detection over conventional MR imaging from $40 \%-60 \%$ to $80 \%-100 \%$, with an almost perfect $\kappa$ $(0.87)$.
The overall conventional MR imaging specificity was higher than that of DWI for detecting skull metastases (97.3\%-100\% versus $83.8 \%-91.9 \%)$. For breast cancer, conventional MR imaging was $100 \%$ specific for both readers. However, DWI was $80 \%-100 \%$ specific, and for lung cancer, conventional MR imaging was again $100 \%$ specific for both, whereas DWI was $76.2 \%-85.7 \%$ specific. The specificity of both imaging techniques for prostate cancer was $100 \%$. For other cancer types, both techniques had similar specificities (90\%-100\% for conventional MR imaging and 100\% for both readers for DWI).

DWI and conventional MR imaging were compared for the detection of focal-versus-diffuse metastasis (Table 3). DWI demonstrated improved detection of focal metastasis relative to conventional MR imaging (57.7\%-61.5\% versus $30.8 \%$ $38 \%)$. Both DWI and conventional MR imaging offered approximately equal sensitivity for detecting diffuse metastasis.

\section{Discussion}

DWI provides important information in patients with cancer outside the CNS not available on conventional MR imaging sequences. In patients being screened for brain metastasis, DWI improved the detection of all types of skull metastasis over conventional MR imaging by $21.1 \%$. When categorized 
Table 3: cMRI versus DWI detection of focal-versus-diffuse skull metastasis

\begin{tabular}{llccc}
\hline MRI Sequence Performed & \multicolumn{2}{c}{ Sensitivity } & & Agreement Beyond \\
on RNBS-Positive Subjects, & $\begin{array}{c}\text { Chance of } \\
n=38\end{array}$ & $\begin{array}{c}\text { Reader } 1 \\
(\%)\end{array}$ & $(\%)$ & Readers \\
\hline cMRl, focal, $n=26$ & $38(10 / 26)$ & $30.8(8 / 26)$ & $0.23(16 / 26)$ & Fair \\
DWI, focal, $n=26$ & $61.5(16 / 26)$ & $57.7(15 / 26)$ & $0.46(19 / 26)$ & Moderate \\
cMRl, diffuse, $n=12$ & $91.7(11 / 12)$ & $66.7(8 / 12)$ & $0.17(7 / 12)$ & Slight \\
DWl, diffuse, $n=12$ & $91.7(11 / 12)$ & $91.7(11 / 12)$ & $1(12 / 12)$ & Perfect \\
\hline
\end{tabular}

Note:- cMRI indicates conventional MR imaging.

by primary tumor type, DWI improved the detection of breast cancer metastasis by $20 \%$, and for lung cancer, by $36.3 \%$. DWI did not improve the detection of prostate metastasis. DWI was most useful for detecting focal metastases, especially in areas with complex anatomy and multiple different normal signal intensities, such as the supraorbital region and the skull base.

DWI likely offers improved detection of lytic skull metastasis compared with conventional MR imaging sequences due to an increased contrast-to-noise ratio. DWI images are exponentially diffusion-weighted and linearly T2-weighted. Lytic metastases are likely very hyperintense on DWI sequences because dense cell packing in highly malignant tumors markedly decreases the diffusion of water ${ }^{8}$ and because lytic metastases are typically hyperintense on T2WI. Furthermore, the background normal skull bones are markedly hypointense: the intradiploic fat is hypointense because echo-planar sequences are typically obtained with fat suppression. Cortical bone is hypointense because there are not enough mobile protons to measure a diffusion coefficient.

DWI is not helpful relative to conventional MR imaging for detecting skull metastases in some circumstances: when the primary lesion is prostate carcinoma or when the skull metastases are diffuse. Prostate metastases are primarily sclerotic. Sclerotic lesions contain increased bone matrix that does not have enough mobile protons to measure a diffusion coefficient. Therefore, similar to adjacent normal bone, prostate metastases are very hypointense on DWI. Diffuse metastases, due to their extensive replacement of normal marrow, are detected with high sensitivity on conventional MR images, and the increased contrast to noise afforded with DWI is not needed for their detection.

The improved detection of skull metastasis with DWI has clinical importance. Given that the skull is the only site of skeletal metastasis in up to $11.6 \%$ of cancer patients, ${ }^{2}$ a diagnostic study with improved sensitivity for skull lesions is a valuable tool in cancer detection in patients undergoing brain MR imaging for indications other than cancer evaluation and who may not have had a previous RNBS. Although many skull metastases are clinically asymptomatic, detection of skull base metastases in the region of cranial nerve foramina is particularly important. DWI may help determine the exact lesion location seen on RNBS that is not evident in retrospect on conventional MR imaging. Early detection of metastases near cranial nerve foramina and of metastases adjacent to dural sinuses may allow early treatment with focal irradiation and preservation of neurologic function. Furthermore, DWI may help differentiate nerve compression due to skull base metastases from nerve injury due to leptomeningeal metastases. In addition, DWI has the potential to be useful in monitoring therapy in patients with infiltrative marrow disease. ${ }^{21}$ For ex- ample, Byun et $\mathrm{al}^{22}$ assessed 23 patients with diffuse vertebral body metastases who clinically improved following radiation therapy to the spine. There was significant decrease in signal intensity on DWI with associated increase in apparent diffusion coefficient values.

Limitations of this study include using RNBS as the gold standard for selecting patients with skull metastasis. The high, but not perfect, sensitivity of the gold standard RNBS for metastatic disease of approximately $92.5 \%{ }^{23}$ excludes from the study those types of metastases not detected by RNBS. We evaluated a relatively small number of bone metastases from each separate primary tumor. It is unclear whether our results for specific tumors will be validated with larger numbers. The specificity of DWI for skull metastasis detection is lower than that of conventional MR imaging; therefore, DWI has a higher false-positive rate. Potential sources of false-positive findings on RNBS and DWI are benign lesions such as fibrous dysplasia. The T2WI and gadolinium-enhanced T1WI were not fatsaturated, a technique that may improve the sensitivity of conventional MR imaging scans for the detection of skull metastases. Our results could depend on lesion size that was not measured. The control subjects were not matched for age or primary malignancy. During the up to 6-week interval between RNBS and MR imaging, a new metastasis could develop. Furthermore, because this is a retrospective study performed over a 6-year time span, the studies were performed with different hardware and software that have gradually improved with time.

\section{Conclusion}

DWI improves the detection of focal skull metastases for breast and lung malignancies. DWI is insensitive for the detection of skull metastases from prostate carcinoma. MR imaging is routinely obtained in cancer patients to detect intracranial metastasis and to detect other important neurologic conditions such as infection and infarction. Furthermore, DWI is cost-effective, requiring only 32 seconds to perform. Therefore, given the clinical relevance of detecting skull metastasis, we believe that DWI should be performed in every cancer patient undergoing brain MR imaging.

\section{References}

1. Tofe AJ, Francis MD, Harvey WJ. Correlation of neoplasms with incidence and localization of skeletal metastases: an analysis of 1,355 diphosphonate bone scans. J Nucl Med 1975;16:986-89

2. Nunan TO, Clarke SE, Coakley AJ, et al. Is the skull view necessary in routine gamma camera bone scanning? Nucl Med Commun 1985;6:37-40

3. Chien D, Kwong KK, Gress DR, et al. MR diffusion imaging of cerebral infarction in humans. AJNR Am J Neuroradiol 1992;13:1097-102

4. Schaefer PW, Grant PE, Gonzalez RG. Diffusion-weighted MR imaging of the brain. Radiology 2000;217:331-45

5. Baur A, Stabler A, Bruning R, et al. Diffusion-weighted MR imaging of bone 
marrow: differentiation of benign versus pathologic compression fractures. Radiology 1998;207:349-56

6. LeBihan DJ. Differentiation of benign versus pathologic compression fractures with diffusion-weighted MR imaging: a closer step toward the "holy grail" of tissue characterization. Radiology 1998;207:305-07

7. Nakagawa K, Sakuma H, Ichikawa Y, et al. Vertebral compression fractures: differentiation between benign and malignant lesions with diffusionweighted single-shot echo planar MR imaging. Eur Radiol 2000;10:154

8. Baur A, Huber A, Ertl-Wagner B, et al. Diagnostic value of increased diffusion weighting of a steady-state free precession sequence for differentiating acute benign osteoporotic fractures from pathologic vertebral compression fractures. AJNR Am J Neuroradiol 2001;22:366-72

9. Spuentrup E, Buecker A, Adam G, et al. Diffusion-weighted MR imaging for differentiation of benign fracture edema and tumor infiltration of the vertebral body. AJR Am J Roentgenol 2001;176:351-58

10. Finelli DA. Diffusion-weighted imaging of acute vertebral compressions: specific diagnosis of benign versus malignant pathologic fractures. AJNR Am J Neuroradiol 2001;22:241-42

11. Herneth AM, Philipp MO, Naude J, et al. Vertebral metastases: assessment with apparent diffusion coefficient. Radiology 2002;225:889-94

12. Falcone $S$. Diffusion-weighted imaging in the distinction of benign from metastatic vertebral compression fractures: is this a numbers game? AJNR Am J Neuroradiol 2002;23:5-6

13. Zhou X, Leeds NE, McKinnon GC, et al. Characterization of benign and metastatic vertebral compression fractures with quantitative diffusion MR imaging. AJNR Am J Neuroradiol 2002;23:165-70

14. Chan JHM, Peh WCG, Tsui EYK, et al. Acute vertebra body compression fractures: discrimination between benign and malignant causes using apparent diffusion coefficients. Br J Radiol 2002;75:207-14

15. Maeda M, Sakuma H, Maier SE, et al. Quantitative assessment of diffusion abnormalities in benign and malignant vertebral compression fractures by line scan diffusion-weighted imaging. AJR Am J Roentgenol 2003;181:1203-09

16. Bammer R, Herneth AM, Maier SE, et al. Line scan diffusion imaging of the spine. AJNR Am J Neuroradiol 2003;24:5-12

17. Raya JG, Dietrich O, Reiser MF, et al. Techniques for diffusion-weighted imaging of bone marrow. Eur J Radiol 2005;55:64-73

18. Castillo M, Arbelaez A, Smith JK, et al. Diffusion-weighted MR imaging offers no advantage over routine noncontrast MR imaging in the detection of vertebral metastases. AJNR Am J Neuroradiol 2000;21:948-53

19. Castillo M. Diffusion-weighted imaging of the spine: is it reliable? AJNR Am J Neuroradiol 2003;24:1251-53

20. McGinn T, Wyer PC, Newman TB, et al. Tips for learners of evidence-based medicine. 3. measures of observer variability (kappa statistic). CMAJ 2004;171:1369-73

21. Herneth AM, Friedrich K, Weidekamm C, et al. Diffusion weighted imaging of bone marrow pathologies. Eur J Radiol 2005;55:74-83

22. Byun WM, Shin SO, Chang Y, et al. Diffusion-weighted MR imaging of metastatic disease of the spine: assessment of response to therapy. AJNR Am J Neuroradiol 2002;23:906-12

23. Hsia TC, Shen YY, Yen RF, et al. Comparing whole body $18 \mathrm{~F}-2$-deoxyglucose positron emission tomography and technetium-99m methylene diphosphate bone scan to detect bone metastases in patients with non-small cell lung cancer. Neoplasma 2002;49:267-71 